\title{
Factors Controlling Pattern Formation in Chemically Amplified Resists at Sub-100 nm Dimensions
}

\author{
W. Hinsberg, F. Houle, G. Wallraff,M. Sanchez, M. Morrison, J. Hoffnagle, \\ Hiroshi Ito, Cattien Nguyen, C. E. Larson, P. J. Brock and G. Breyta \\ IBM Research Division, Almaden Research Center \\ 650 Harry Road, San Jose, CA USA 95120
}

\begin{abstract}
At minimum feature dimensions below $100 \mathrm{~nm}$, the required dimensional tolerances for pattern formation in integrated circuit fabrication approach the length scales of the molecular components and processes typically found in a resist film. This paper summarizes recent experimental work aimed at an improved understanding of photoacid diffusion and line-edge roughness, two key factors that influence dimensional control in chemically amplified resists.
\end{abstract}

Keywords: aerial image contrast, line-edge roughness, chemically amplified resist, diffusion, interferometric lithography, simulation

\section{Introduction}

The development of photoresist technology for the manufacture of integrated circuits with feature sizes of 100 nanometers and below presents a formidable challenge. In this range, acceptable dimensional tolerances begin to approach the length scales of the molecular components and processes in the resist film Linewidth variations that are permissible at larger feature sizes may be completely unacceptable at these small dimensions

Two factors are considered to be significant potential sources of linewith variations in the nanoscale regime. Random fluctuations in the width of a resist feature, termed line-edge roughness (LER), is one of these[1][2]. Possible sources of LER include the molecular weight and molecular weight distribution of the resist polymer and the molecular structure of resist components[3][4], inhomogeneity in the distribution of resist components within the film[5], statistical effects influencing film dissolution[6] and intrinsic properties of the imaging chemistry[7].

The second factor, diffusion of the photogenerated catalytic acid species, is specific to chemically amplified (CA) resists, which are anticipated to be used in future semiconductor fabrication regardless of the imaging technology [8]. The effect of photoacid diffusion has been a concern since the earliest days of chemically amplified resist development. It was believed by some that excessive diffusion would seriously limit the utility of these resist systems at dimensions much lower than 1 micron in size[9]. While this clearly is not the case - devices with $180 \mathrm{~nm}$ features are now being manufactured and sub 100 $\mathrm{nm}$ features have been printed with a variety of exposure techniques - a clear understanding of the true contribution of acid diffusion to linewidth variations remains elusive. In the absence of this understanding a variety of approaches have been employed in the hopes of minimizing diffusion effects. These include the use of bulky photoacids[10] or the incorporation of basic additives to quench the acid as it diffuses out of the exposed area of the film [11]. Since acid diffusion in polymers is a thermally activated process, the use of imaging chemistry which requires low post exposure bake temperatures has also been utilized [12] [13].

Understanding and quantifying the influence of resist composition and process conditions on the magnitude of these effects is important both from the standpoint of photoresist design and with regard to the development of accurate resist models for use in lithographic simulation programs. In this report we summarize recent work aimed at an improved understanding of these factors. 


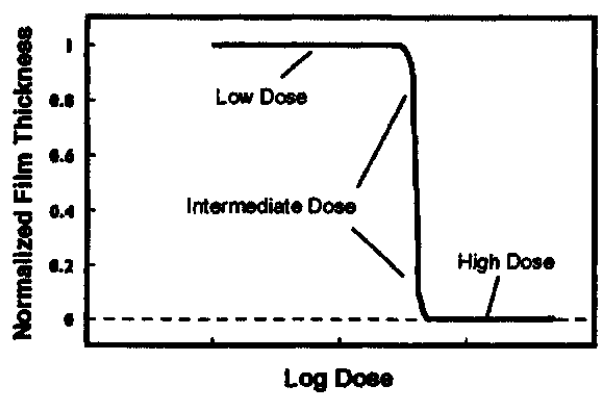

(a)

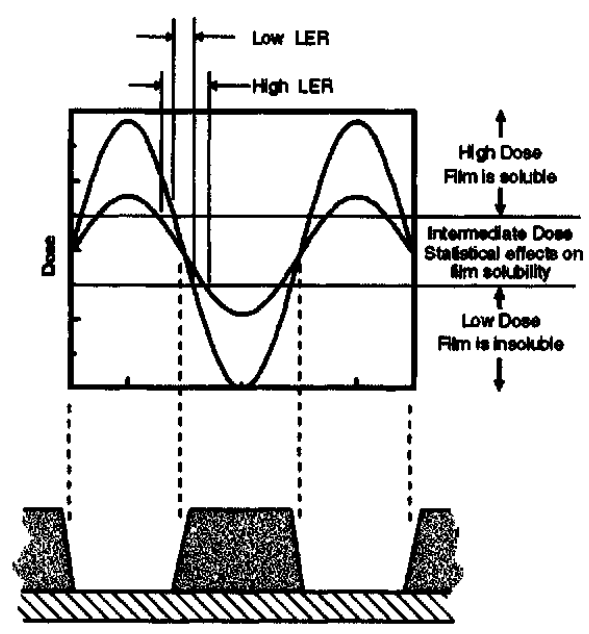

(b)

Figure 1. Schematic illustrating the effect of aerial image contrast on LER in a single layer positive-tone resist[15]; (a) contrast curve typical of a high-contrast positive-tone CA resist; (b) relationship between the light image projected onto a resist film and the developed resist pattern.

\section{Line-edge Roughness as a Function of Aerial Image Contrast}

In a detailed study of LER in top-surface imaging (TSI) resists by MIT's Lincoln Laboratory[14], a correlation between LER and surface roughness of exposed silylated and developed TSI resists was established. The MIT study showed that unsilylated (high exposure dose) and heavily silylated (low exposure dose) surfaces exhibit little texture, but that films with intermediate levels of exposure, and therefore silylation, show pronounced surface roughness. This was interpreted as stemming from statistical effects in the intermediate dose region that lead to spatially nonuniform silicon incorporation at the nanoscale. This result led to the prediction that LER in TSI resists is linked to aerial image contrast (AIC), and the MIT group has reported TSI imaging results consistent with this prediction.

Deep-UV interferometric lithography (IL) provides the unique ability to form sub- $100 \mathrm{~nm}$ resist structures while precisely and independently controlling dose, pitch and AIC during exposure. In an earlier report, we employed IL to directly examine the effect of varying AIC on the imaging properties of a single-layer photoresist[15]. We found that, for that material, LER increased with decreasing AIC, behavior analogous to the TSI results cited above. In the present work, we have extended that original study, with the goal of identifying resist attributes that influence LER.

Figure 1 schematically illustrates how LER can be related to AIC in the case of a single layer photoresist. Figure 1(a) shows a schematic plot of normalized film thickness remaining after development versus log exposure dose (termed a contrast curve) that typifies the dose-response behavior of high-contrast positive-tone CA resists. At low doses the film is essentially insoluble, while at high doses the film rapidly and completely dissolves. In the transition region between these extremes, however, the solubility behavior changes rapidly with dose. Typical values for the slope of the curve in this region (termed the contrast $\gamma$ ) are in the range 6-10; in other terms, a $25-50 \%$ change in dose is sufficient to convert the resist film from an insoluble to a soluble state. It is precisely the dissolution behavior in this region of the exposed image that determines the acuity of the resist line edge.

Figure 1(b) relates the intensity of the exposing light image to the final resist pattern, and shows the relationship of the intermediate dose region of Figure 1(a) to the developed resist image. Those portions of the resist film exposed at the maximum of the image are rendered highly soluble, while those portions exposed at the minimum remain essentially insoluble. In the intermediate dose transition region, the solubility switches between these extremes. If a single layer CA resist 
(a)
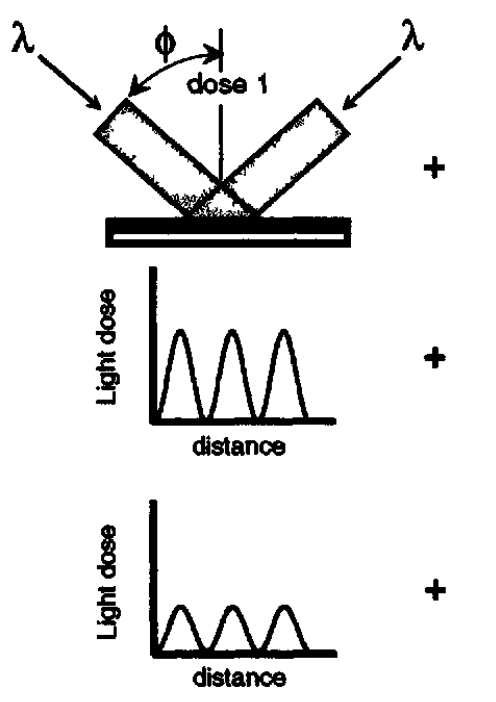
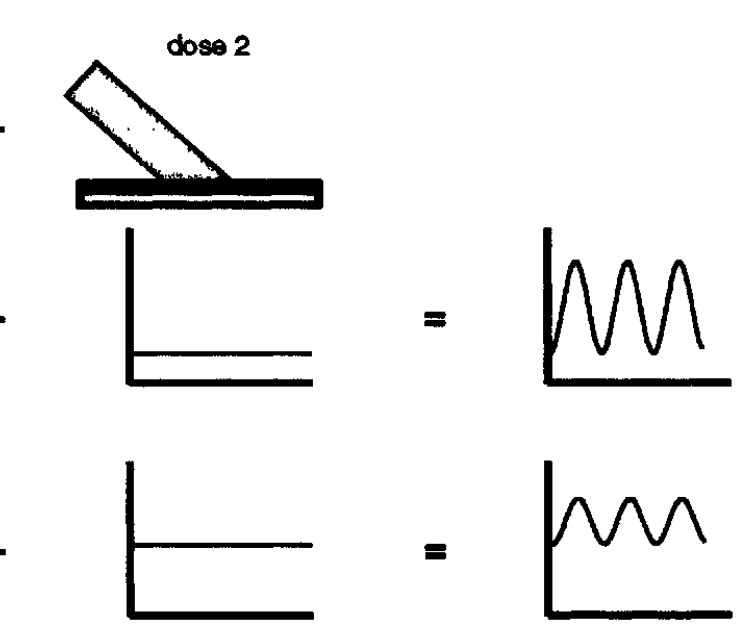

(b)

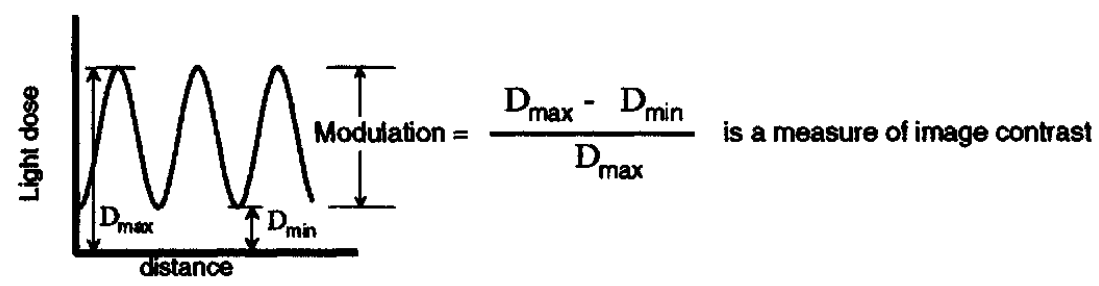

Figure 2. Interferometric lithography. (a) a dual exposure protocol for the synthesis of aerial images of controlled contrast is illustrated. By combining an imaging IL exposure and a flood exposure, any desired integrated dose and image contrast can be realized; (b) diagram illustrating the definition of image modulation used in the study .

were to possess spatially inhomogeneous solubility properties in this intermediate dose range, for example due to nonuniform dispersion of the photoacid generator in the film[5], nanoscale statistical variations in the extent of acid-catalyzed deprotection[7], or statistical effects on the solubility of polymer chains [6], then the developed line-edge will replicate that inhomogeneity as LER. Figure 1(b) illustrates how the contrast of the aerial image would influence the apparent LER in such a case. Two light images are depicted, with differing contrast between light and dark areas. With a high contrast light image, the intermediate region is rapidly traversed, minimizing statistical effects of solubility. With the low contrast image, a larger fraction of the image period is located within this transition region, and the wider transition through the region of statistical variation on solubility leads to increased LER.

\subsection{Experimental Method.}

Our instrumentation for carrying out interferometric lithography has been described in detail elsewhere[15]. We utilize the sinusoidal standing wave pattern of light intensity produced at the intersection of two coherent light beams to print periodic line-space patterns in resist films (Figure 2). The period of the pattern is determined by the wavelength of light and the angle of intersection of the beams, and the minimum resolution of the method can far exceed conventional projection photolithographic exposure tools using the same wavelength. For example, using light beams with a wavelength of $257 \mathrm{~nm}$ incident at an angle $\phi$ of 80 degrees, a line-space array of period $135 \mathrm{~nm}$ is attainable, roughly half that with a state-of-the-art $248 \mathrm{~nm}$ projection tool.

With balanced beams, the IL image is intrinsically $100 \%$ modulated. This modulation can 
be varied in a controlled manner to any desired value by carrying out a dual exposure sequence [15]. Each site is first exposed with a two-beam imaging exposure, followed with a flood exposure using a single beam. By appropriate selection of the two doses, any desired image contrast can be achieved(Figure 2).

In the experiments described here, a diverse set of single layer photoresists was imaged using IL. The image pitch was held constant at $500 \mathrm{~nm}$ (nominal $250 \mathrm{~nm}$ lines $/ 250 \mathrm{~nm}$ spaces). The exposure dose was optimized individually for each resist system, and was chosen so that equal line-space dimensions were formed upon exposure at $100 \%$ AIC. The AIC was then systematically varied while holding the total integrated dose constant at this value. Scanning electron microscopy of the relief images, followed by analysis using an imaging analysis software package, provides a numeric estimate of the magnitude of LER. The analySIS ${ }^{\oplus}$ software image analysis package from Soft Imaging Systems was used with their optional linewidth module. The line-edge was found using a noise filtered algorithm with a $10 \%$ threshold. The line-edge roughness is reported as the standard deviation of the linewidth.

The resist materials set was selected to comprise representatives from a number of dissimilar resist classes. Positive and negative tone chemically amplified (CA) resists were included to examine the influence of different imaging chemistries. Those negative-tone CA resists include materials designed to develop in aqueous base (where dissolution follows an acid-base reaction between the polymer and the developer components) and in organic solvents (where the resist film is dissolved without a chemical reaction with the developer) to probe the role of the development mechanism. Resists with identical composition but varying polymer molecular weights were examined to test the effect of polymer chain length on LER. Finally, a typical DNQ-novolac resist (based on non-CA imaging chemistry) was included to determine if LER is a consequence of the chemical amplification mechanism. The materials and process conditions are listed in Table 1.

\subsection{Results}

SEM images of a typical AIC experiment are shown in Figure 3. These images are formed in

Table 1. Materials and processing conditions.

\begin{tabular}{|c|c|c|c|c|c|}
\hline PHOTORESIST & ARC & $\begin{array}{c}\text { PAB } \\
\left({ }^{\circ} \mathrm{C} / \text { time }\right)\end{array}$ & $\begin{array}{c}\text { DOSE } \\
\left(\mathrm{mJ} / \mathrm{cm}^{2}\right)\end{array}$ & $\begin{array}{c}\text { PEB } \\
\left({ }^{\circ} \mathrm{C} / \text { time }\right)\end{array}$ & Developer/time \\
\hline \multicolumn{6}{|l|}{ Positive-tone $C A$} \\
\hline Shipley UVII-HS & BARL-900 & $130 / 60$ sec. & 2.5 & $140 / 90$ sec. & Shipley CD-26/30 sec. \\
\hline UV5 & BARL-900 & $130 / 60 \mathrm{sec}$. & 2.86 & $140 / 90 \mathrm{sec}$. & Shipley CD-26/30 sec. \\
\hline UV6 & BARL-900 & $130 / 60 \mathrm{sec}$. & 2.6 & $140 / 90 \mathrm{sec}$. & Shipley CD-26/30 sec. \\
\hline \multicolumn{6}{|c|}{ Negative-tone/Aqueous base developed CA } \\
\hline CGR2205 & AR3-600 & $95 / 60 \mathrm{sec}$. & 1.9 & $95 / 60 \mathrm{sec}$. & $0.14 \mathrm{~N}$ Shipley CD-26/60 sec \\
\hline Shipley UVN30 & AR3-600 & $110 / 60 \mathrm{sec}$. & 2.5 & $95 / 60 \mathrm{sec}$. & Shipley CD-26/30 sec \\
\hline a) $\mathrm{CGR}(2.5 \mathrm{~K} \mathrm{MW})$ & AR3-600 & $95 / 60$ sec. & 1.43 & $95 / 60 \mathrm{sec}$. & $0.10 \mathrm{~N}$ Shipley CD-26/60 sec \\
\hline a) CGR (8K MW) & AR3-600 & $95 / 60 \mathrm{sec}$. & 1.1 & $95 / 60 \mathrm{sec}$. & $0.13 \mathrm{~N}$ Shipley CD-26/60 sec \\
\hline a) CGR (15K MW) & AR3-600 & $95 / 60$ sec. & 1.25 & $95 / 60 \mathrm{sec}$. & $0.19 \mathrm{~N}$ Shipley CD-26/60 sec \\
\hline \multicolumn{6}{|c|}{ Negative-tone/Organic solvent developed CA } \\
\hline SU8 & & $85 / 3 \mathrm{~min}$. & 0.8 & $90 / 3 \mathrm{~min}$. & b) PGMEA/45 sec. \\
\hline \multicolumn{6}{|l|}{ Non-Acid Catalyzed } \\
\hline c)Clariant AZ7905 & XHRi-16 & $110 / 2 \mathrm{~min}$. & 136 & $135 / 90 \mathrm{sec}$. & $1: 4 \mathrm{AZ} 400 \mathrm{~K}: \mathrm{H}_{2} \mathrm{O} / 60 \mathrm{sec}$. \\
\hline
\end{tabular}




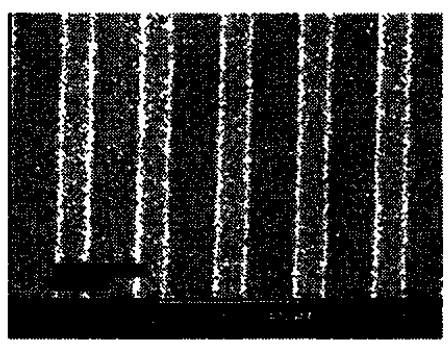

$100 \%$

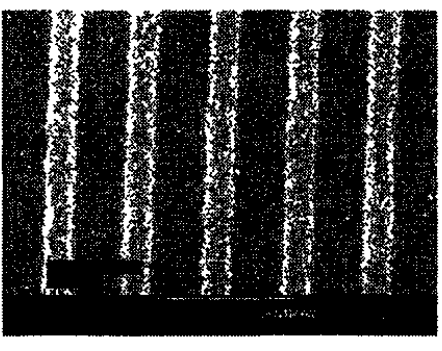

$61 \%$

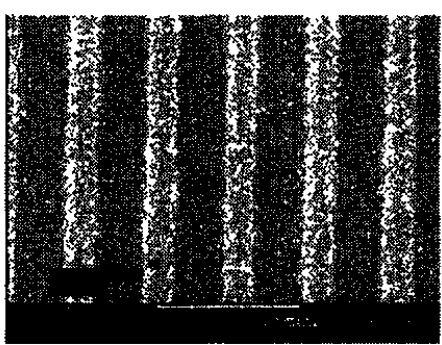

$49 \%$

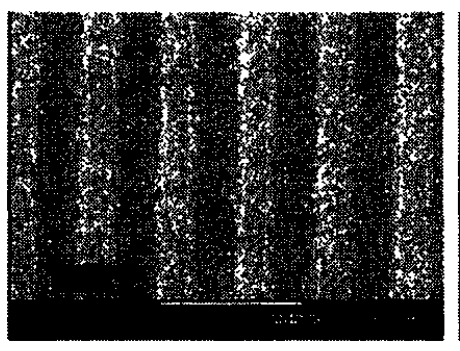

$36 \%$

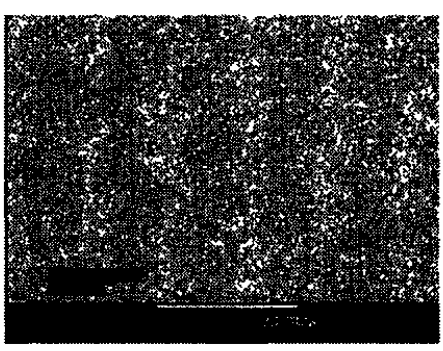

$10 \%$

Figure 3. Scanning electron micrographs of resist images obtained when a film of Shipley UVII-HS positive-tone chemically amplified photoresist is exposed to light images of varying image contrast using $257 \mathrm{~nm}$ interferometric lithography. The linewidth is unchanged with image contrast but the LER increases sharply as the image contrast degrades.

Shipley UVII-HS photoresist, after exposure to the aerial images of varied contrast $\left(3.9 \mathrm{~mJ} / \mathrm{cm}^{2}\right.$ integrated dose), followed by postexposure processing. Note that the linewidth remains essentially constant in this series, as one would expect, since the dose near the line edge does not change appreciably. The images demonstrate visually that LER increases as AIC decreases, consistent with our earlier prediction for resists that exhibit statistical effects on solubility.

This same trend is seen in all photoresists studied to date. The graph in Figure 4 compares the values of LER, derived by software image analysis, for resists and process conditions listed in Table 1. At $100 \%$ AIC, all systems produce images with distinct, sharp edges, and have very low values of LER that are the same within experimental error. The average measured LER at $100 \%$ AIC is $2-7$ $\mathrm{nm}$, or about 1-4 percent of the resist linewidth. This residual roughness is on the order of the molecular size of polymers typically used in resist formulations (estimated by the radius of gyration for polymers with molecular weights in the range of 5000 to 100,000 ). The residual LER value likely includes some uncertainty attributable to the SEM imaging and analysis. At values of AIC in the range typically found in high resolution projection lithography near the resolution limit ( $60-70 \%$ AIC), definite differences in the materials emerge. The relation between LER and AIC varies widely among the materials studied.

A pairwise comparison of selected curves in Figure 4 provides information on the possible origins of LER. Both AZ7905 (a positive-tone diazonaphthoquinone-based resist) and UVII-HS (a positive-tone CA photoresist) show LER responses of similar magnitude. While this does not rule out statistical effects in the deprotection chemistry as a factor in LER[7], such a mechanism cannot be operant in the AZ7905 material. In another comparison, UVN30 and SU8 [16][17] are both negative-tone resists functioning on the basis of acid-catalyzed crosslinking. UVN 30 is designed for development in aqueous alkaline solution while SU8 is developed in an organic solvent. Both materials show similar increases in LER with decreasing AIC. While this does not rule out statistical effects in aqueous base development as a factor in LER, such a mechanism cannot be operant with the SU8 material. Finally, a comparison of the set of three positive-tone CA resists (UVII-HS, UV5 and UV6) to one another and to the set of three negative-tone 


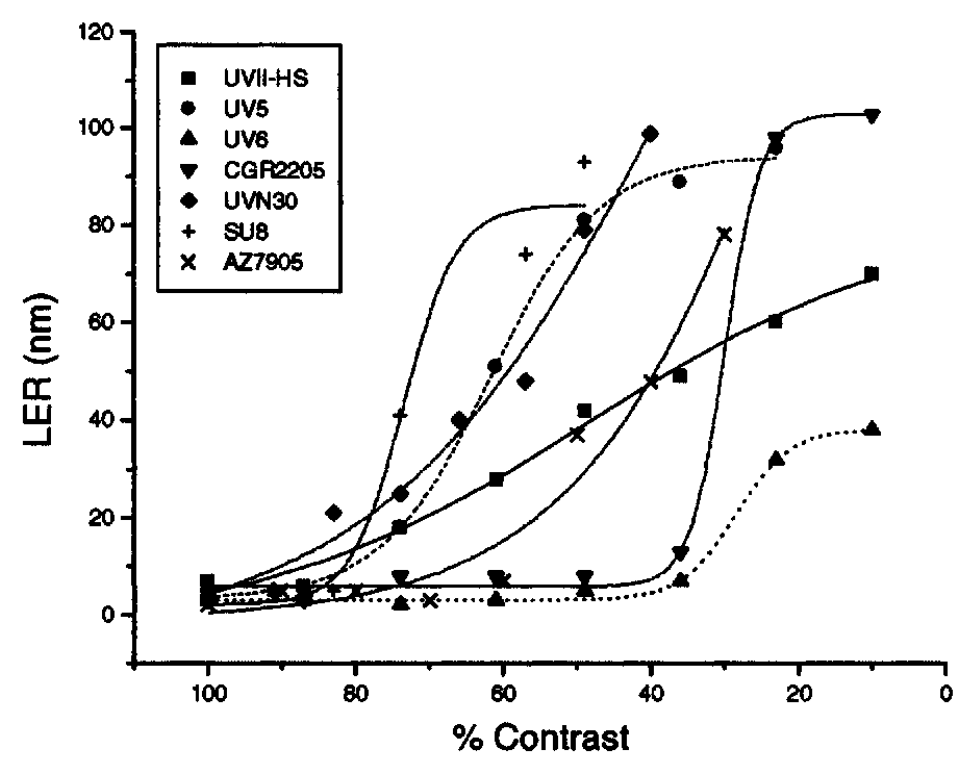

Figure 4. Line-edge roughness as a function of decreasing contrast for different resist materials

CA resists (CGR, UVN30 and SU8) demonstrates that both sets encompass similar wide ranges of LER response.

These comparisons lead to the conclusion that no single isolated factor governs LER. The common element among all the resist materials we have examined is that each exhibits high resist contrast, that is, each undergoes highly nonlinear changes in film solubility with exposure dose. While high resist contrast is a key requirement for advanced semiconductor lithography, in general the chemical and physical origins for the nonlinear dissolution response at present are not well understood. We speculate that LER is an undesirable consequence of the threshold dissolution behavior that is the basis of high resist contrast.

Figure 4 suggests that the resist materials composition is a significant factor governing the relation between AIC and LER. We have carried out an initial assessment of how polymer molecular weight influences that interrelation in CGR resist. CGR is a negative-tone, CA deep-UV photoresist based on poly(hydroxystyrene) (PHOST), the crosslinking agent tetramethoxymethylglycoluril, and an N-hydroxy sulfonate ester photoacid generator[18]. Three CGR formulations of identical composition were prepared, each using a near-monodisperse PHOST of different molecular weight $\left(M_{n}\right.$ values of 2500,8000 and 15000). The impact of AIC of LER for these three formulations was assessed using our standard IL protocol. Since the speed of dissolution of PHOST is a steep function of molecular weight, the concentration of developer was adjusted for each formulation so that unexposed films of each CGR variant dissolved at the same rate. All other process conditions were held constant.

Analysis of the resulting images reveals that molecular weight has a pronounced effect on the relation between LER and AIC. This is best illustrated by direct visual examination of SEM images (Figure 5). At 100\% AIC, all three resist formulations exhibit the same behavior, producing relief images with essentially no LER. When AIC is decreased to $60 \%$, the low molecular weight resist (2.5K) shows little change, but the formulations with midrange $(8 \mathrm{~K})$ and high $(15 \mathrm{~K})$ molecular weight polymer show sharply increased LER. This trend is consistent with an earlier study of the influence of polymer molecular weight on LER in negative-tone electron beam resists based on bis-azide crosslinkers [3][4]. The midrange CGR formulation displays increased nano-edge roughness at $60 \%$ AIC, while the high molecular weight CGR resist displays increased nano-edge roughness of the same magnitude accompanied by the appearance of microbridging between features. The microbridging appears to be near the level of the substrate and does not contribute to the nano-edge roughness at 


\section{$100 \%$ Contrast}

(2.5K)

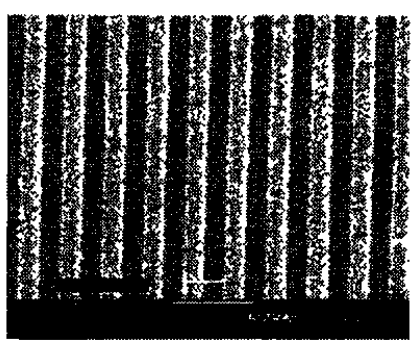

\section{$60 \%$ Contrast}

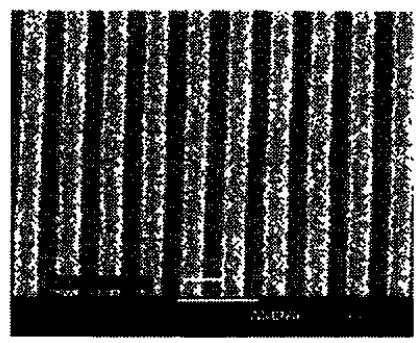

LER:
(8K)
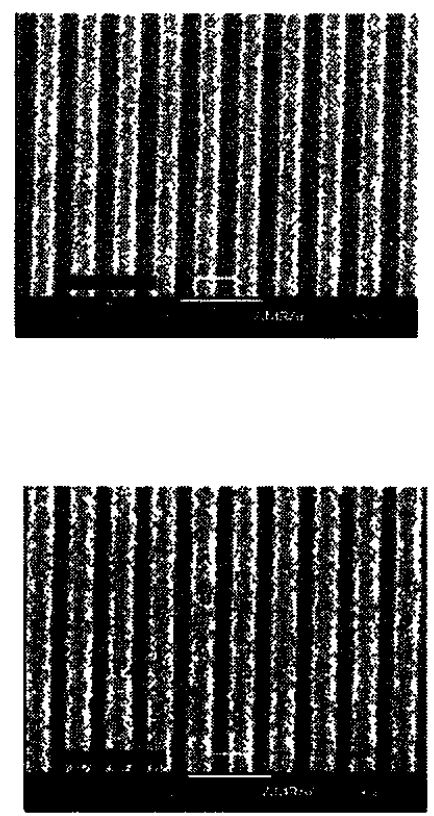

$60 \mathrm{~nm}$
(15K)
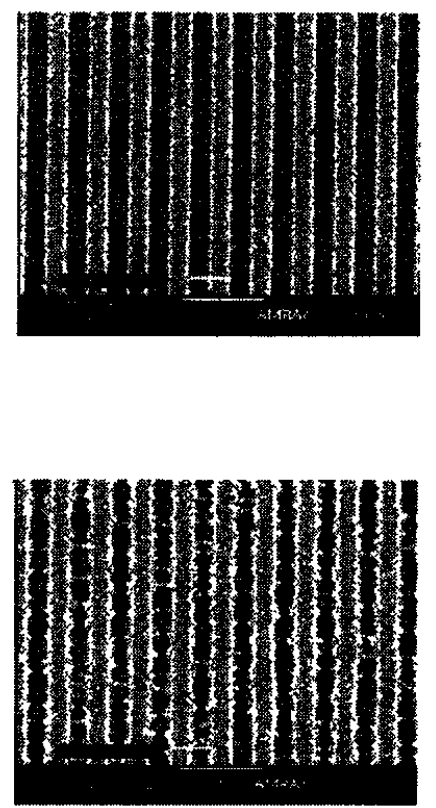

$50 \mathrm{~nm}$

Figure 5. SEM micrographs of molecular weight effect on line-edge roughness. The first row shows the line-space array at $100 \%$ contrast for each of the three molecular weights. The second row shows the change in line-edge roughness with a decreased contrast of $60 \%$.

the top of the resist image. The image analysis software parameters are set such that microbridging is excluded in the numeric estimate of LER. In our results, the onset of nano-edge roughness and microbridging occur at different polymer molecular weights suggesting that they are independent phenomena. Microbridging has been identified in similar negative-tone CA resist chemistries[19] [20] where its severity was shown to increase with higher polymer molecular weights and/or with stronger developer hydroxide concentration. We are unable to distinguish between these two possibilities with the CGR data now at hand.

\section{Experimental Measurement of Acid Diffusion}

A number of reports have described methods for the characterization of acid diffusion and linewidth changes in resist polymers. Experiments intended to quantify photoacid diffusion by ion conductivity measurements[21][22], by correlations with acid volatilization[23], and absorption of acid vapor[24], STM measurements[25], and recently by laser confocal microscopy[26] have been described. In our view none of the approaches described to date offer the optimum method for measuring acid diffusion.

\subsection{Experimental Method.}

We have developed a simple technique for generating acid gradients. It relies on surface exposure wherein acid is generated in a thin sublayer of the polymer film near the air-film interface. This can be readily accomplished, for example, by exposing a phenolic DUV resist with $193 \mathrm{~nm}$ radiation, where the high absorbance of the aromatic polymer serves to limit light penetration in the resist film. The deprotection front can then be tracked as it moves down through the resist film (Figure 6). The advantage of this approach is that the acid profile is determined in a simple way by the absorbance of the polymer and the quantum yield of photoacid generator (PAG). The technique measures changes over large areas and does not require imaging of the reacting film at high spatial 


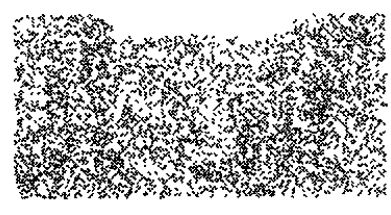

Initial Photoacid profile

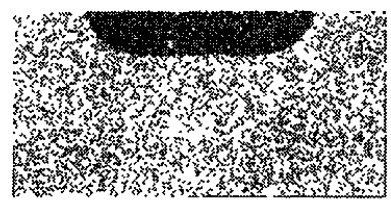

Deprotection profile (low diffusion case)

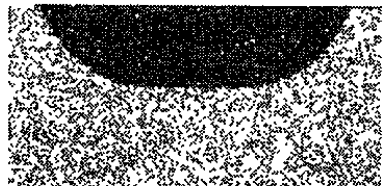

Deprotection profile

(high diffusion case)

Figure 6. Surface exposure or TSI method for generating photoacid profiles

resolution. Measuring the extent of deprotection as a function of time during the post-expose heating step provides quantitative information on the kinetic and diffusion parameters of the resist. Photoacids with higher diffusion coefficients will give higher rates of deprotection as they diffuse into the resist film. Other one-dimensional approaches based on laminating or spin-casting pairs of dissimilar films have been described previously[27] [28] [29].

Poly(tert-butoxycarbonyloxystyrene) polymer (PTBOCST) was prepared according to the published procedure[30]. The bis tert-butylphenyliodonium (hereafter abbreviated at TBI) perfluoroalkylsulfonate photoacid generators were prepared via standard metathesis procedures[31]. In a typical experiment, a film of PTBOCST resist containing 0.045 molal TBI-PFBS (TBI-perfluorobutanesulfonate) was spin-coated from a solution in propylene glycol methyl ether acetate onto a one inch diameter undoped silicon wafer and post-apply baked at $130{ }^{\circ} \mathrm{C}$ for five minutes to give a final thickness of $1.2 \mu \mathrm{m}$. The film was then exposed to a dose of $70 \mathrm{~mJ} / \mathrm{cm}^{2}$ at $193 \mathrm{~nm}$ wavelength using an ISI microstepper, and the exposed wafer was placed on the preheated hot stage of a Nicolet Nic-Plan infrared microscope operating in transmission mode. Infrared absorption spectra were recorded at predetermined periods during thermolysis. During the measurement, the resist-coated wafer was isolated from the atmosphere using an infrared-transparent cover plate. The extent of deprotection as a function of time was calculated from changes in the TBOC carbonyl absorbance at $1760 \mathrm{~cm}^{-1}$.

Diffusion parameters were extracted from the dynamic data using a modified version of a stochastic chemical kinetics simulator[32][33]. To do so requires knowledge of the kinetic parameters for the resist deprotection chemistry which were independently obtained using an analogous protocol on films with a uniform distribution of acid. Films with uniform acid distribution were prepared by blanket exposure to a high exposure dose (200 $\mathrm{mJ} / \mathrm{cm}^{2}$ ) at $254 \mathrm{~nm}$ on ARC coated wafers; this insures a uniform distribution of acid throughout the resist film. Quantitation of photogenerated acid was performed using bromphenol blue titration in the standard fashion [34]. The optical properties of the resist films were determined using a Cary $\mathbf{4 0 0}$ spectrophotometer and a Woolam spectroscopic ellipsometer.

\subsection{Results}

The dynamic measurement technique can be used to compare the effects of counterion size on the extent of deprotection in PTBOCST films. Figure 7 displays the extent of deprotection versus bake time for top-surface-exposed PTBOCST films, each containing the same molar concentration of a different TBI perfluoroalkylsulfonate PAG, where the size of the counterion is varied from $106 \AA^{3}$ (for trifluoromethanesulfonate), to 240 $\AA^{3}$ (for perfluorobutanesulfonate), to $340 \AA^{3}$ (for the $\mathrm{C}_{8} \mathrm{~F}_{15} \mathrm{SO}_{3}{ }^{-}$anion) [35]. Due to their structural similarity all three PAGs are expected to have nearly identical quantum yields for acid generation and to produce very strong acids having similar $\mathrm{pK}_{\mathrm{a}}$ values; the former were verified by spectrophotometric titration measurements. Note that the extent of deprotection is significantly higher for TBI trifluoromethanesulfonate (TBIT) than for the two larger PAGs.

Diffusion parameters can be extracted from rate data of the type shown in Figure 7 provided that both the kinetic parameters for the resist reactions and the concentration and distribution of photoacid in the surface exposed film are known. 
Film Composition vs Time for Surface-Exposed PTBOCST/PAG Films

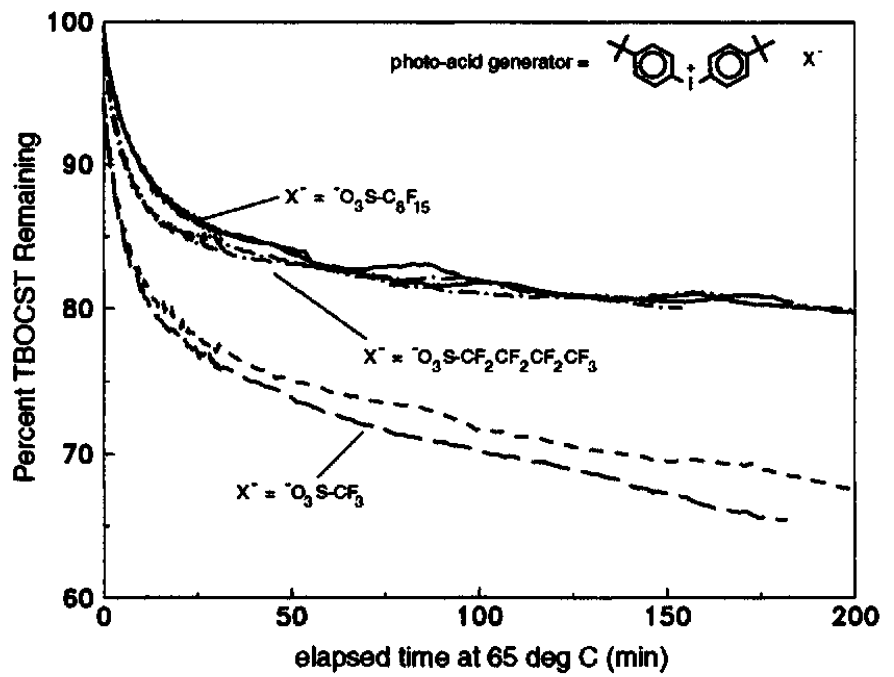

Figure 7. Extent of deprotection measured for PTBOCST films containing TBI perfluoroalkylsulfonate PAGs of differing anion size. The curves show the extent of deprotection a post-expose bake temperature of $65^{\circ} \mathrm{C}$ after exposure with $193 \mathrm{~nm}$ light at a dose of $70 \mathrm{~mJ} / \mathrm{cm}^{2}$.

This will be illustrated for the PTBOCST/ TBI-PFBS) resist system.

The acid catalyzed deprotection of PTBOCST is described using the following mechanism

$$
\begin{aligned}
& \text { pTBOCSTH } \\
& \text { pTBOCSTH }^{+} \rightarrow \mathrm{pHOST} \rightleftharpoons \mathrm{pHBOCST}^{+} \\
& \text {pTBOCST } \rightarrow \text { pHOST }
\end{aligned}
$$$$
\text { pTBOCST }+2 \text { pHOST } \rightarrow 3 \text { pHOST }
$$

\section{SCHEME 1}

This scheme was previously established for the reaction between PTBOCST and strong acids [36] and is consistent with the mechanism for specific acid catalysis that occurs in solution[37]. Step (1) represents the equilibrium between the protonated TBOC protecting groups and phenolic pendent groups. Step (2) is the acid induced cleavage step in this reaction. Steps (3) and (4) describe direct uncatalyzed thermolysis of PTBOCST[36], reaction pathways that can become significant at high temperature and/or high levels of conversion. Kinetic parameters for steps (1) and (2) were extracted from the measured deprotection kinetics of PTBOCST films containing $2.5 \mathrm{wt} \%$ TBI-PFBS, uniformly exposed to a high dose at $254 \mathrm{~nm}$ and are listed in Table II. These are in good agreement with previously reportedvalues[36].

The initial photoacid distribution was calculated for an incident intensity of $70 \mathrm{~mJ} / \mathrm{cm}^{2}$ from the absorbance of the film at $193 \mathrm{~nm}(\mathrm{OD}=$ 13), using a quantum yield of 0.2 . The quantum yield value was derived by fitting the deprotection versus time curve for the PTBOCST/TBIT system at $65^{\circ} \mathrm{C}$ where diffusion is very slow. The acid is distributed in the film as a uniform layer from the film-air interface to a depth of about $160 \mathrm{~nm}$ thick, with a weak tail extending to about $260 \mathrm{~nm}$ depth

For the purposes of simulation, the polymer film is considered to be comprised of a collection of discrete cells or layers with a specified initial

\begin{tabular}{|c|c|c|c|c|c|}
\hline $\begin{array}{l}\text { REACTION } \\
\text { STEP }\end{array}$ & CONSTANT & $A\left(\sec ^{-1}\right)$ & $E_{n}(\mathrm{kcal} /$ mole $)$ & $\Delta \mathrm{H}(\mathrm{kcal} / \mathrm{mole})$ & $\Delta S$ (cal/mole) \\
\hline (1) & $\mathrm{K}_{\mathrm{eq}, 1}$ & - & - & -8.8 & -25.1 \\
\hline (2) & $\mathbf{k}_{\mathrm{d}, 2}$ & $1.4 \times 10^{18}$ & 28.67 & - & - \\
\hline (3) & $\mathbf{k}_{\mathrm{d}, 3}$ & $7.3 \times 10^{12}$ & 32.7 & - & - \\
\hline (4) & $\mathbf{k}_{d, 4}$ & $6.3 \times 10^{11}$ & 29.9 & - & - \\
\hline
\end{tabular}
composition determined by the initial photoacid

Table 2.Rate coefficients used in simulations 


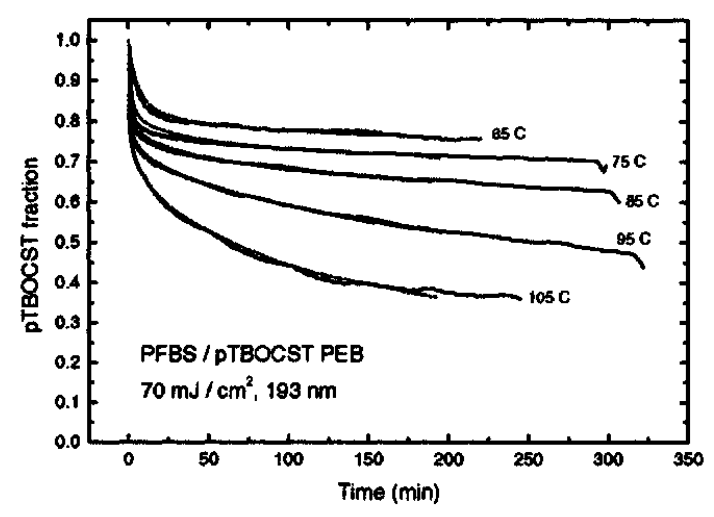

(a)

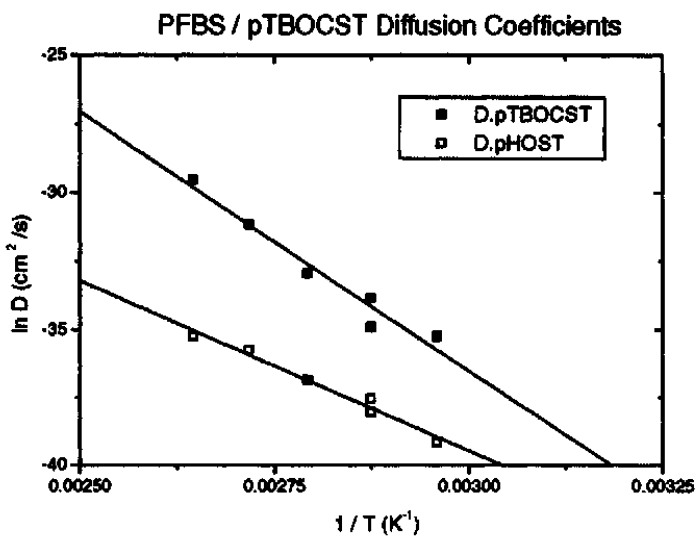

(b)

Figure 8. (a) Comparison of simulated (dashed lines) and experimental spectrophotometric curves (solid lines) for PTBOCST/TBI-PFBS deprotection from $65-105^{\circ} \mathrm{C}$; (b) Arrhenius plot of best-fit diffusion coefficients for movement of acid through PTBOCST and through PHOST.

distribution. In this example the PTBOCST film was represented by a $1 \mathrm{~cm}^{2}$ stack of 120 layers, each ten $\mathrm{nm}$ thick. In each layer, both the acid-catalyzed and the direct thermolysis deprotection pathways shown in Scheme 1 are enabled. As the conversion of PTBOCST to PHOST proceeds, the thickness of each layer shrinks according to the fractional molar densities of the two polymeric components. This volume change allows the concentrations of the polymers to be properly renormalized during the simulation to maintain accurate kinetics.

Each of the 120 layers is connected to its neighbors by a bi-directional diffusion path which allows migration of acid through the film. A salient characteristic of the deprotection of PTBOCST is that the deprotection reaction is accompanied by a change in polarity of the polymer from non-polar to polar. This environmental change is expected to have a strong effect on acid diffusion kinetics because they involve migration of charged species. Thus, acid diffusion is modeled as a process involving two coexisting pathways: migration through PTBOCST and migration through PHOST. The diffusion steps are represented by a site-exchange process in order to avoid explicitly treating migration of protons in the polymer, a process that is poorly understood. The diffusion rate is the standard Fickian form. The calculations yield complete concentration versus. time predictions for each layer that are then converted into total PTBOCST disappearance curves for comparison to the experimental data.

Best-fit simulations are compared with experimental data in Figure 8(a). Overall, the fits are quite close over the entire temperature range, indicating that the coupled reaction-diffusion model provides a good description of the chemistry. An Arrhenius plot for the two diffusion coefficients derived from the data analysis is shown in Figure 8(b). The diffusion coefficient for perfluorobutanesulfonic acid (HPFBS) in PHOST is described by the Arrhenius parameters

$$
D_{\text {PHOST }}=0.16 \exp (-24.9 / \mathrm{RT}) \mathrm{cm}^{2} / \mathrm{sec} \text {. }
$$

The diffusion coefficient for HPFBS in PTBOCST is described by the Arrhenius parameters

$$
D_{\text {PTвосST }}=6.3 \times 10^{8} \exp (-37.6 / \mathrm{RT}) \mathrm{cm}^{2} / \mathrm{sec}
$$

where the gas constant $R$ is in units of $\mathrm{kcal} \mathrm{deg}^{-1}$ mole $^{-1}$ and the temperature $T$ is in degrees $\mathrm{K}$. Error bars derived from standard deviations to the linear fits are approximately $10^{2}$ for $D_{0}$ and $3 \mathrm{kcal} / \mathrm{mol}$ for the $\mathrm{E}_{\mathrm{a}}$. The corresponding diffusion coefficients at $85^{\circ} \mathrm{C}$ are $1 \times 10^{-16}$ and $7 \times 10^{-15} \mathrm{~cm}^{2} / \mathrm{sec}$ for pHOST and PTBOCST respectively. These coefficients are significantly smaller than the most closely corresponding resist diffusion coefficients reported previously; these earlier values range from $10^{-12}$ to $10^{-14}$ [38][39]. Our values are orders of magnitude slower than diffusion coefficients for polymer diffusants which are nonionic and which would not be expected to interact strongly with the polymer matrix, for example by protonation. One example is the diffusion of camphorquinone in poly(methylmethacrylate) at $150^{\circ} \mathrm{C}$ where a diffusion coefficient of $3.55 \times 10^{-11} \mathrm{~cm}^{2} / \mathrm{sec}$ has been reported[40].

The quantitative kinetic model we have determined allows us to consider how the deprotected polymer profile compares to the initial 


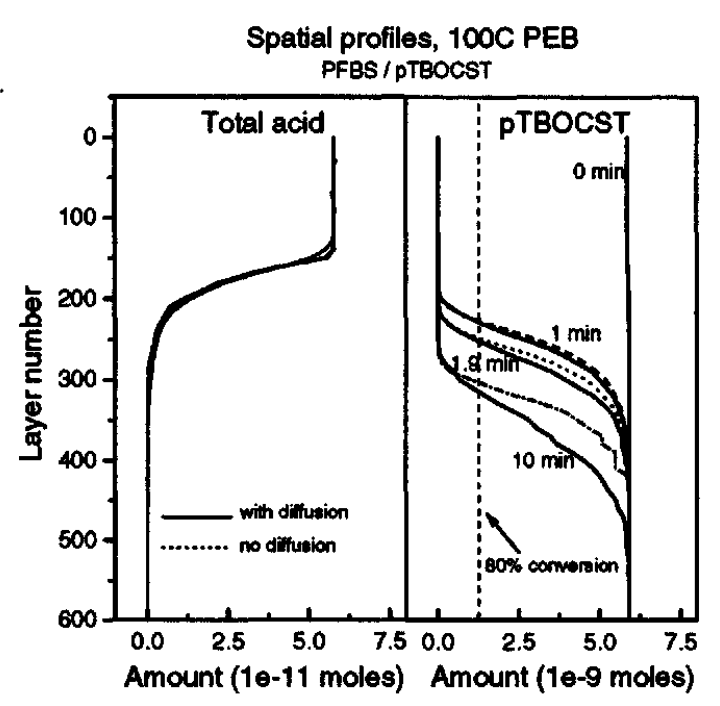

(a)

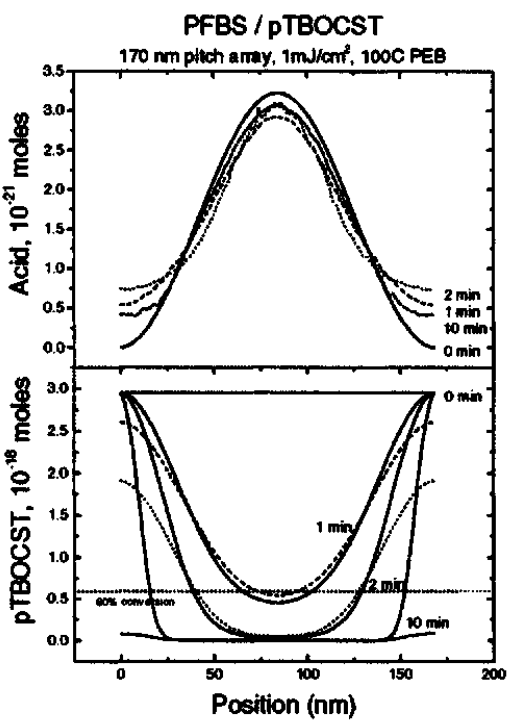

(b)

Figure 9. (a) Cross sectional profile for surface exposed PTBOCST/TBI-PFBS film. Plotted on the left is the acid concentration as a function of initial layer depth (i.e. layer 200 is at an initial depth of $200 \mathrm{~nm}$ in the film). On the right is shown the corresponding penetration depth of the deprotected polymer. Spatial profiles with and without diffusion are shown in each case;(b) simulated profiles for $170 \mathrm{~nm}$ pitch, dense line-space array structure. The upper graph represents the acid profile as a function of bake time, the lower graph the corresponding profile of the remaining PTBOCST polymer

latent image of photogenerated acid as the post-exposure bake process proceeds. This provides direct information on the role of diffusion in limiting image resolution in this resist system. We have calculated profiles for PEB at $100^{\circ} \mathrm{C}$. Figure 9(a) shows profiles for acid and PTBOCST in the $193 \mathrm{~nm}$ top-down exposure experiment for the first 10 minutes with diffusion both enabled and disabled (i.e. where the diffusion coefficients are set to zero.).

This corresponds to printing sparse structures at the quarter micron scale. A line marking the $80 \%$ PTBOCST-PHOST conversion point is shown as an indication of what the approximate developed thickness would be for the two cases. It is evident that there is little if any change in the acid profile curing a two minute post exposure bake. Nonetheless, there is significant movement of the boundary of deprotected polymer whether diffusion is allowed or not. Interestingly, the final developed film thickness would be only slightly larger if there were no diffusion of the acid. At ten minutes, on the other hand, differences become marked even though the acid profile is essentially static. This indicates that macroscopic movement of acid is not necessary for catalyzed deprotection to occur: sporadic escape of acid is sufficient to spread the image because of the pronounced chemical amplification.

Interesting differences are found for dense line-space array structures at $170 \mathrm{~nm}$ pitch (Figure 9(b)). This simulation is for uniform $254 \mathrm{~nm}$ exposure at $1 \mathrm{~mJ} / \mathrm{cm}^{2}$. In this case relaxation of the initial acid image is more pronounced as expected for a higher resolution structure. Also, population of initially acid-free regions is significant compared to the top-down exposure because of the very small confined volume the acid can migrate to. As in the case of the surface exposed system there is significant outward movement of the deprotected region. At short times, comparable to a standard PEB, this spread cannot be attributed to diffusion. Diffusion effects only become apparent at significantly longer times under these low dose (low acid concentration) conditions.

\section{SUMMARY}

\subsection{Line-edge Roughness}

Using UV interferometric lithography combined with a two-step exposure protocol, we 
have assessed the influence of aerial image contrast on line-edge roughness for a diverse set of high contrast photoresist materials. At maximum aerial image contrast $(100 \%$ intensity modulation of the aerial image), all materials in this set display very low line-edge roughness ( $2 \%$ of the linewidth). As the aerial image contrast is decreased, in all cases the magnitude of line-edge roughness increases. The precise behavior ranges from a nearly linear increase in roughness to a step-function increase depending on the resist material. Resist composition appears to be a significant factor governing this behavior, though no specific class of resist chemistry emerges from our data as offering a distinct advantage in this regard. To demonstrate how the methodology described here can be applied to the systematic study of resist composition effects on line-edge roughness, the polymer molecular weight was systematically varied in a negative-tone chemically amplified resist formulation. Our imaging results indicate that polymer molecular weight can be a significant factor influencing edge roughness. At maximum aerial image contrast, the magnitude of line-edge roughness was low and independent of molecular weight. As aerial image contrast is decreased, edge roughness in the low molecular weight formulation remains small, while midrange and high molecular weight formulations display significant and equivalent increases in edge roughness. In addition, the high molecular weight formulation exhibits microbridging as aerial image contrast is decreased; the onset of these features with molecular weight, and their positioning on the resist structures suggest that microbridging and nano-edgeroughness are independent effects.

\subsection{Photoacid Diffusion.}

Measuring the extent of reaction of surface exposed films is a useful technique for assessing the effects of photoacid diffusion in chemically amplified resists. Experiments on PTBOCST films exposed at $193 \mathrm{~nm}$ show clear differences attributable to PAG size when photoacid generators of comparable acidity and quantum yield are qualitatively compared.

Diffusion coefficients have been extracted from deprotection rate data on surface imaged PTBOCST/TBI-PFBS films. Stochastic simulation of the reacting resist film employing a model based on the site exchange of protonated resist components yields different diffusion coefficients for PTBOCST and PHOST. Diffusion through the polar, hydrogen-bonded PHOST matrix was found to be appreciably slower than diffusion in PTBOCST. Both sets of coefficients fall outside the range of reported values and are consistent with acid mobility lower than previously thought. Simulations of the relative motions of photoacid and the PHOST reaction product show that only very low concentrations of acid are required to effect significant levels of deprotection at large penetration depths.

\section{ACKNOWLEDGMENTS}

We would like to thank J. Opitz and Debra Fenzel-Alexander for their technical assistance, and W. Brunsvold, A. Renaldo, J. Gelorme, and N. LaBianca for providing resist materials and processing recommendations. This work has been supported, in part, by National Science Foundation Grant DMR-9400254 (CPIMA).

\section{REFERENCES}

1. J. Hutchinson, V. Rao, G. Zhang, A. Pawloski, C. Fonseca, S. Holl, S. Das, C. Henderson, D. Wheeler, Proc. Soc. Photo-Opt. Instr. Eng., 3333, 165 (1998).

2. E. Shiobara, D. Kawamura, K. Matsunaga, T. Koike, Sh. Mimotogi, T. Azuma, Y. Onishi, Proc. Soc. Photo-Opt. Instr. Eng., 3333, 313 (1998).

3. T. Yoshimura, H. Shiaishis, J. Yamamoto, and S. Okazaki, Jpn. J. Appl. Phys., 32, 6065 (1993).

4. J. Yamamoto, S. Uchino, T. Hattori, T. Yoshimura, and F. Murai, Jpn. J. Appl. Phys., 35, 6511 (1996).

5. D. McKean, R. Allen, P. Kasai, U. Schaedeli, and S. MacDonald, Proc. Soc. Photo-Opt. Instr. Eng., 1672, 94 (1992).

6. P. Tsiartis, C. Henderson, L. Flanagin, W. Hinsberg, I. Sanchez, R. Bonnecaze, and G. Willson, Macromolecules, 30, 4656 (1997).

7. T. Ushirogouchi, K. Asakawa, M. Nakase, and A. Hongu, Proc. Soc. Photo-Opt. Instr. Eng., 2438, 609 (1995).

8. 1997 National Technology Roadmap for Semiconductors, published by the Semiconductor Industry Association, San Jose, California, 1997.

9. A. Umbach, A. Broers, R. Koch, C. Willson and R Laibowitz, IBM J. Res. Dev. 32, 454 (1988). 
10. M. Cronin, M. Adams, T. Fedynyshyn, J. Georger, J. Mori, R. Sinta, J. Thackery, Proc. Soc. Photo-Opt. Instr. Eng, 2195, 214 (1994).

11. K. Asakawa, T. Ushirogouchi and M. Nakase, Proc. Soc. Photo-Opt. Instr. Eng, 2438, 563 (1995).

12. W. Huang, R. Kwong, A. Katnani, and M. Khojasteh, Proc. Soc. Photo-Opt. Instr. Eng, 2195, 37 (1994).

13. H. Schacht, N. Muenzrl, P. Falcigno, H. Holzwarth, and J. Schneider, J. Photopolym. Sci. Technol. , 9, 445 (1996).

14. S. Palmateer, S. Cann, J. Curtin, S. Doran, L. Eriksen, A. Forte, R. Kunz, T. Lyszczarz, M. Stern and C. Nelson, Proc. Soc. Photo-Opt. Instr. Eng., 3333, 634 (1998).

15. W. Hinsberg, F.A. Houle, J. Hoffnagle, M. Sanchez, G. Wallraff, M. Morrison, and S. Frank, J. Vac. Sci. Technol. B, 16, 3689 (1998).

16. K. Stewart, M. Hatzakis, J. Shaw, D. Seeger and E. Neumann, J. Vac. Sci. Techn. B, 7(6), 1734 (1989).

17. K. Lee, N. LaBianca, S. Rishton, S. Zolgharnain, J. Gelorme, J. Shaw and T. Chang, J. Vac. Sci. Techn. B, 13(6), 1734 (1995).

18. W. Brunsvold, W. Conley, J. Gelorme, R. Nunes, R. Sooriyakumaran, S. Holmes and J. Sturtevant, Proc. Soc. Photo-Opt. Instr. Eng., 2195, 329 (1994).

19. J. Thackeray, G. Orsula and M. Denison, Proc. Soc. Photo-Opt. Instr. Eng., 2195, 152 (1994).

20. L. Linehan, R. Smith, J. Dorn, J. Fahey, W. Moreau, G. Spinillo, E. Puttlitz and J. Collins, Proc. Soc. Photo-Opt. Instr. Eng., 2438, 211 (1994).

21. J. Nakamura, H. Ban, K. Deguchi, and A. Tanaka, A. Jpn. J. Appl. Phys., 10, 6065 (1991).

22. T. Fedynyshyn, J. Thackeray, J. Georger and M. Denison, J. Vac. Sci. Technol. (B) , 12, 3888 (1994).

23. J. Thackeray, M. Denison, T. Fedynyshyn, D. Kang and $R$. Sinta, in Microlectronics Technology, Polymers for Advanced Imaging and Packaging, ACS Symposium Series 614; E. Reichmanis, C. Ober, S. MacDonald, T.
Iwayanagi and T. Nishikubo, eds., American Chemical Society, Washington, DC. 1995, 84.

24. K. Mueller, W. Koros, C. Mack, and C. Willson, Proc. Soc. Photo-Opt. Instr. Eng., 3049, 706 (1997).

25. F. Perkins, E. Dobisz and C. Marrian, J. Vac. Sci. Technol. (B) , 11, 2597 (1993).

26. P. Zhang, S. Webber, J. Mendenhall, J. Beyers and K. Chao, Proc. Soc. Photo-Opt. Instr. Eng., 3333, 794 (1998).

27. K. Asakawa, T. Ushirogouchi and M. Nakase, Proc. Soc. Photo-Opt. Instr. Eng., 2438, 563 (1995).

28. S. Uchino, J. Yamamoto, S. Migitaka, K. Kojima, M. Hashimoto, F. Murai, and H.;Shiraishi, J. Photopolym. Sci. Technol. , 11, 555 (1998).

29. Sakamizu, T.; Arai, T.; Yamaguchi, H.; Shiraishi, H. Proc. Soc. Photo-Opt. Instr. Eng., 3049, 448 (1997).

30. J. Frechet, E. Eichler, H. Ito and G. Willson, Polymer, 24, 995 (1983).

31. G. Breyta, R. DiPietro, H. Ito and D. Hofer, U. S. Patent 5,585,220 (December 17, 1996).

32. W. Hinsberg and F. Houle, U. S. Patent $5,826,065$ (October 20, 1998).

33. Information on the stochastic simulation of chemical kinetics and the CKS kinetics simulation program package can be found at web site http://www. almaden.ibm.com/st/msim.

34. Buhr, G.; Dammel R.; Lindey, C., PMSE Proceedings, 61, 269 (1989).

35. Approximate molecular volumes were determined from molecular mechanics calculations (PCMODEL) on minimized structures (MM2 based).

36. G. Wallraff, J. Hutchinson, W. Hinsberg, F. Houle, P. Seidel, R. Johnson and W. Oldham, J. Vac. Sci. Technol. B, 12, 3857 (1994).

37. T. Lowery and K. Richardson, in Mechanism and Theory in Organic Chemistry; 3rd Ed. Harper and Row: New York, 1987; Chapter 7.

38. T. Itani, H. Yoshino, S. Hashimoto, M. Yamana, N. Samoto and K. Kasama, J. Vac. Sci. Technol. B, 14, 4226 (1996), 
39. J. Thackeray, T. Adams, M. Cronin, M. Denison, T. Fedynyshyn, J. Georger, J. Mori, G. Orsula and R. Sinta, J. Photopolym. Sci.
Technol., 7, 619 (1994).

40. J. Zhang, B. Yu and C. Wang, J. Phys. Chem., 90, 112 (1986). 